\title{
Measurements and Corrections of the Recycler Lattice at Fermilab
}

\author{
Meiqin Xiao
}

Abstract: Obit Response Matrix (ORM) Fit method has been successfully used to calibrate linear optics at Recycler Ring at Fermilab. The linear model of the Recycler optics ring has been significantly improved. Based on the build-up model, lattice measurement of the Recycler ring has been done several times, each after some magnets move and the tunes change. Large beta-wave( 20\%) has been found in horizontal plane after the working point was moved from $(0.424,0.434)$ to $(0.456,0.467)$ for the reason of lowering the beam instabilities. The source of the beta-wave, and the correction will be presented in this paper. In addition, we found an easy way to extend the tuning range in the recycler lattice. A new application program for adjusting the tunes operationally was introduced and the measured results will be presented.

\section{Introduction}

Obit Response Matrix (ORM) Fit method has been successfully used to calibrate linear optics at Recycler Ring at Fermilab [1]. Based on differential orbit measurement, the linear model of the Recycler optics has been significantly improved. With the build-up linear modeling, the measurements of the Recycler lattice have been done several times. The first orbit response measurement was done in Oct. 2005 and a full set of data consisting of roughly 150 orbits was taken on Feb.25, 2006. The data was then split into 3 sets which were analyzed separately. The found 3 sets of quadrupole errors were different but the values of beta-functions resulting from application of these errors were within $2 \%$ from each other, shown in Fig.1. Also it was found that there is a little bata-wave although the relative beta function errors are up to $40 \%$ in horizontal and $20 \%$ in vertical planes compared to those of the designed lattice.

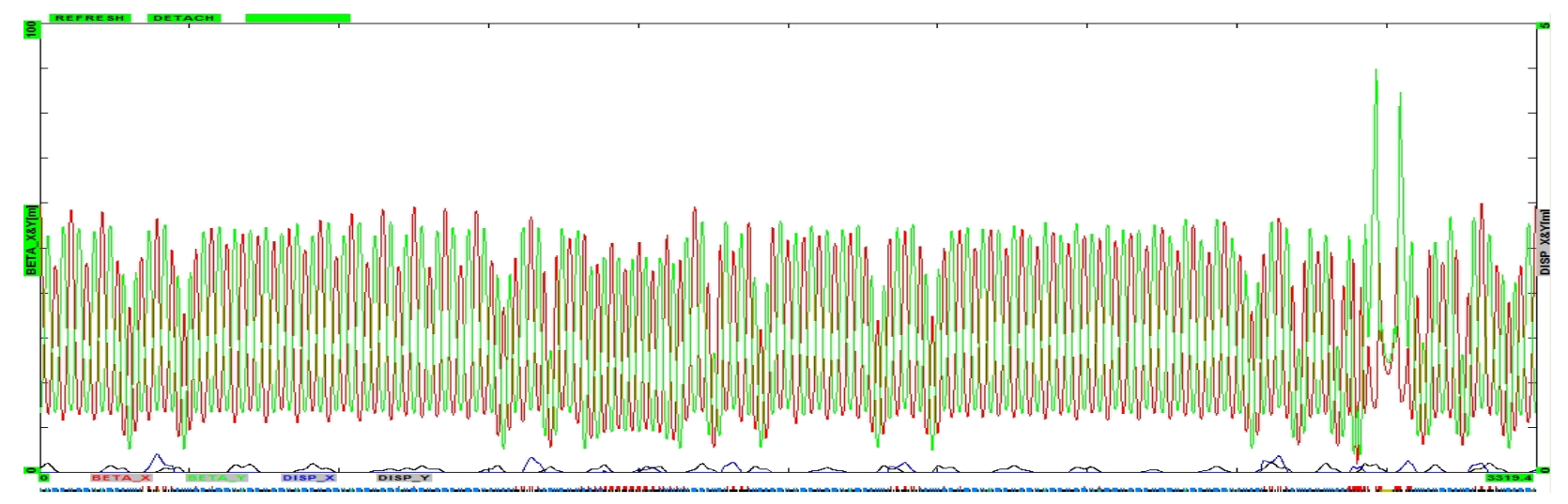

Fig.1 Beta-functions obtained from differential orbit measurement at working point of $(25.424,24.434)$

*Work supported by U.S. Department of Energy under contract No. DE-AC02-76CH03000.
\#meiqin@fnal.gov 
During 2006 summer shut down, we did some magnet move in the ring so that the corrector's strength can be reduced after orbit smooth. We also moved the recycler ring's working point from $(0.424,0.434)$ to $(0.456,0.467)$ primarily for the reason of lowering the beam instability during the mining process. With all these change, we repeat the orbit response measurements with a larger number of the differential orbits taken and found a very large bata-wave in horizontal plan, shown in Fig.2.

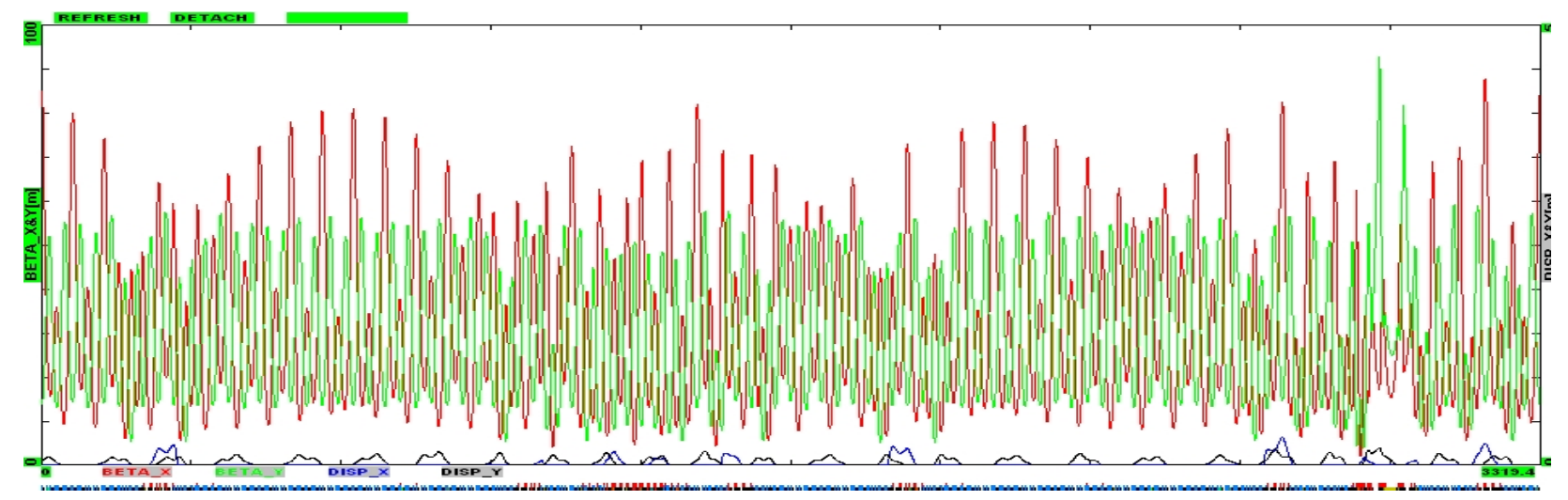

Fig.2 Beta-functions obtained from differential orbit measurement at working point of $(25.455,24.464)$ before TROMBONE program ungraded.

The source of the beta-wave was track down to the phase trombone straight section. The Recycler Ring is an $8-\mathrm{GeV}$ fixed energy ring using permanent magnets. Instead of distributing remotely adjustable quadrupoles around the ring, 9 pairs of independently power supplied adjustable quadrupoles are located in RR-60 straight section, called phase trombone section [2]. The principle of the trombone section is to adjust the phase advances, i.e. tunes in the ring, but keeping the Beta-functions unchanged at two ends, shown in Fig.3 at MRK601, MRK609.

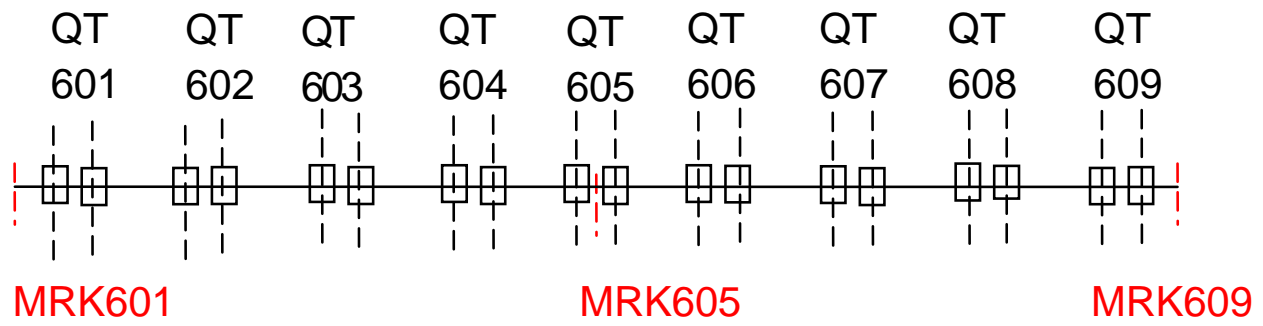

Fig.3 Phase trombone section in the Recycler ring

A phase trombone program has been used operationally for tuning up the betatron tunes. In March 2004, the phase trombone program was migrated once based on designed lattice functions of Recycler Ring [3]. Currently the trombone section is segmented into 5 families to maintain a symmetrical structure as follows:

$$
\left\{\begin{array}{l}
\text { QT 601_I }=\text { QT 609_I }=\text { QBPT 0_I } \\
\text { QT 602_I }=\text { QT 608_I }=\text { QBPT1_I } \\
\text { QT 603_I }=\text { QT 607_I }=\text { QBPT 2_I } \\
\text { QT 604_I }=\text { QT 606_I }=\text { QBPT3_I } \\
\text { QT 605_I }=\text { QBPT 4_I }
\end{array}\right.
$$


By adjusting these circuits, a tune variation of up to \pm 0.06 in horizontal and 0.18 in vertical plane is attainable. Obviously, this program which was based on designed lattice beta-functions is not proper. Table 1 listed the beta-functions calculated from designed lattice and from the build-up model. We can see that the differences are significant. For old working point, the adjustments of the current of 9 quads are relatively small, the beta function leak from the trombone section must be small so that we don't see beta-wave in the rest of the ring. However, at new working point near 0.45 , the beta-leak is severe. Therefore, it is necessary to update the trombone program based on measured beta-functions.

Table 1 beta-functions calculated from designed lattice and from the build-up model

\begin{tabular}{|l|c|l|l|l|l|l|l|l|}
\hline & \multicolumn{3}{|l|}{ Beta-functions @MRK601 } & \multicolumn{3}{l|}{ Beta-functions@MRK609 } \\
\cline { 2 - 9 } & $\beta_{\mathrm{x} 1}$ & $\beta_{\mathrm{y} 1}$ & $\alpha_{\mathrm{x} 1}$ & $\alpha_{\mathrm{y} 1}$ & $\beta_{\mathrm{x} 1}$ & $\beta_{\mathrm{y} 1}$ & $\alpha_{\mathrm{x} 1}$ & $\alpha_{\mathrm{y} 1}$ \\
\hline $\begin{array}{l}\text { Calculated from Designed } \\
\text { lattice }\end{array}$ & 10.103 & 46.958 & -0.098 & 0.014 & 10.114 & 46.367 & 0.008 & 0.012 \\
\hline $\begin{array}{l}\text { Calculated from build-up } \\
\text { model obtained from ORM } \\
\text { measurement }\end{array}$ & $\mathbf{8 . 7 3 3}$ & $\mathbf{4 8 . 0 1 6}$ & $\mathbf{- 0 . 0 9 9}$ & $\mathbf{0 . 0 4 7}$ & $\mathbf{8 . 6 8 3}$ & $\mathbf{4 7 . 8 1 5 2}$ & $\mathbf{- 0 . 0 6 3}$ & $\mathbf{0 . 0 3 3}$ \\
\hline
\end{tabular}

\section{Matching conditions}

We know that each of 9 pairs of quadrupoles in RR-60 straight section has their own power supply. In principle, each of them can be adjusted independently as long as keeping the conditions that the Twiss parameters at the two ends of the straight section unchanged.

We know the changes of the tunes in $x$ and $y$ planes are as follows:

$$
\Delta v_{x, y}= \pm \frac{1}{4 \pi} \sum_{i} \int_{0}^{L} \beta_{x, y}\left(s_{i}\right) k\left(s_{i}\right) d s_{i}
$$

$k\left(s_{i}\right)$ is the strength of $i^{\text {th }}$ quadrupole, $\beta_{x, y}\left(s_{i}\right)$ is the beta-function of the quadrupole.

On the other hand, we have

$$
\left(\begin{array}{l}
\boldsymbol{\beta} 2 \\
\boldsymbol{\alpha} 2 \\
\boldsymbol{\gamma} 2
\end{array}\right)=\left(\begin{array}{ccc}
\boldsymbol{M}_{11}^{2} & -2 \boldsymbol{M}_{11} \boldsymbol{M}_{12} & \boldsymbol{M}_{12}^{2} \\
-\boldsymbol{M}_{11} \boldsymbol{M}_{21} & \boldsymbol{M}_{11} \boldsymbol{M}_{22}+\boldsymbol{M}_{12} \boldsymbol{M}_{21} & -\boldsymbol{M}_{12} \boldsymbol{M}_{22} \\
\boldsymbol{M}_{21}^{2} & -2 \boldsymbol{M}_{21} \boldsymbol{M}_{22} & \boldsymbol{M}_{22}^{2}
\end{array}\right)\left(\begin{array}{l}
\boldsymbol{\beta} 1 \\
\boldsymbol{\alpha} 1 \\
\boldsymbol{\gamma} 1
\end{array}\right)
$$

For both $x$ and $y$ planes. where $M_{11}, M_{12}, M_{21}$ and $M_{22}$ are the elements of the transfer matrix $M$ of the straight section, which are also the functions of the quadrupole strengths. We know $\gamma$ is a function of $\alpha$ and $\beta$, we actually only get two independent equations in each plane. Totally, we get 6 independent equations. 
To calculate the transfer matrix $\boldsymbol{M}$ of the straight section, first we output transfer matrices from code OPTIM [4] between two trim quads, and take each quad as thin element, with integrated strength $(k L)$ $\approx 10^{-3}$, as follows:

$$
M_{q}=\left[\begin{array}{cccccc}
\cos \left(\sqrt{k_{x}} L\right) & \frac{1}{\sqrt{k_{x}}} \sin \left(\sqrt{k_{x}} L\right) & 0 & 0 & 0 & 0 \\
-\sqrt{k_{x}} \sin \left(\sqrt{k_{x}} L\right) & \cos \left(\sqrt{k_{x}} L\right) & 0 & 0 & 0 & 0 \\
0 & 0 & \cosh \left(\sqrt{k_{x}} L\right) & \frac{1}{\sqrt{k_{x}}} \sinh \left(\sqrt{k_{x}} L\right) & 0 & 0 \\
0 & 0 & \sqrt{k_{x}} \sinh \left(\sqrt{k_{x}} L\right) & \cosh \left(\sqrt{k_{x}} L\right) & 0 & 0 \\
0 & 0 & 0 & 0 & 1 & \frac{L}{\beta_{s}^{2} \gamma_{s}^{2}} \\
0 & 0 & 0 & 0 & 0 & 1
\end{array}\right] \approx\left[\begin{array}{cccccc}
1 & 0 & 0 & 0 & 0 & 0 \\
-\boldsymbol{k L} & 1 & 0 & 0 & 0 & 0 \\
0 & 0 & 1 & 0 & 0 & 0 \\
0 & 0 & \boldsymbol{k} \boldsymbol{L} & 1 & 0 & 0 \\
0 & 0 & 0 & 0 & 1 & 0 \\
0 & 0 & 0 & 0 & 0 & 1
\end{array}\right]
$$

and then concatenate the transfer matrix for whole section

$$
M=M_{d 20} \cdot M_{q 609} \cdot M_{d 19} \cdot M_{q 609} \cdot M_{d 18} \cdots M_{q 601} \cdot M_{d 2} \cdot M_{q 601} \cdot M_{d 1}
$$

where $\mathbf{M}_{\mathrm{d} 1}, \mathbf{M}_{\mathrm{d} 2}, \ldots \mathbf{M}_{\mathrm{d} 20}$ are the matrices of drift space between two quads, $\mathbf{M}_{\mathrm{q} 601}, \mathbf{M q}_{602}, \ldots \mathbf{M}_{\mathrm{q} 609}$ are the matrices of the quads 601,602 to 609 . Note that each quadrupole is split into two pieces in the machine.

\section{Console application program TROMBONE upgrade}

A test program was written in Mathematica. The transfer matrix for whole section is first concatenated in the program as given in Equation (4), and then the equations are linearized so that only first order terms of $i^{\text {th }}$ quadrupole strength $k_{i}$ are kept. The general equations are obtained for keeping 9 variables in the role, as follows:

$\left[\begin{array}{ccccccccc}-26.0506 & 831.49 & 59.5737 & -749.714 & -27.5116 & \mathbf{8 6 8 . 0 3 8} & 36.4185 & -773.266 & -12.0946 \\ 17.5588 & 23.5745 & -18.766 & -23.7522 & 17.585 & 8.67871 & -19.3967 & -10.1126 & 17.3344 \\ 2304.8 & -756.055 & -1738.19 & 833.257 & 1243.97 & -829.713 & -458.44 & \mathbf{8 8 1 . 6} & \mathbf{6 7 . 0 3 8 8} \\ -81.5639 & -8.81639 & 85.9938 & 6.52632 & -91.2513 & -3.92004 & 93.7124 & 1.34981 & -95.9362 \\ 1.41081 & 7.9777 & 1.55587 & 7.25293 & 1.41061 & 8.0431 & 1.55596 & 7.193 & 1.38011 \\ -7.64076 & -1.41846 & -7.5213 & -1.45272 & -7.60481 & -1.41309 & -7.53389 & -1.46471 & -7.61725\end{array}\right]\left[\begin{array}{c}K_{1} \\ K_{2} \\ K_{3} \\ K_{4} \\ K_{5} \\ K_{6} \\ K_{7} \\ K_{8} \\ K_{9}\end{array}\right]=\left[\begin{array}{c}-0.00203665 \\ 0.000291693 \\ -0.156029 \\ -0.000528379 \\ \Delta v_{\mathbf{x}} \\ \Delta v_{\mathbf{y}}\end{array}\right]$

It turns out to be a least-squares problem, which can be solved by SVD (Singular Value Decomposition). There are five cases for the conditions of matching and constrains, listed as follows. Note that $K$ in the following equations represents the integrated strength of the quadrupole,

$$
K=k L=0.00297 /(m \cdot A m p) \times L \times I
$$

Where $I$ is circuit current, $L$ is the length, $L=0.3048 \mathrm{~m}$.

For a given tune changes, we have tested 5 different cases, including

(1) 4 equations, 5 families (symmetric): The beta-functions at two ends are not guaranteed unchanged 
(2) 6 equations, 5 families (symmetric): it Can not get expected tunes!!

(3) 6 equations, 6 families --- unique solutions. However, there always have one or two circuits current exceed the limits (6.5Amps)

(4) 6 equations, 7 families $(\mathrm{K} 1, \mathrm{~K} 2, \ldots, \mathrm{K} 5, \mathrm{~K} 6, \mathrm{~K} 8)$ : there still has one of the circuit current exceeds the limit.

(5) 6 equations, 9 families $(\mathrm{K} 1, \mathrm{~K} 2, \ldots, \mathrm{K} 9)$ : all the currents are reasonably, evenly distributed and the beta-functions at two ends can be guaranteed unchanged. For example, given tune change of $\Delta v_{\mathrm{x}}$ $=0.0385, \Delta v_{\mathrm{y}}=0.042$, the currents of 9 circuits are as follows(unit in Amps):

$\mathrm{I} 1, \mathrm{I} 2, \ldots, \mathrm{I} 9=(-1.300,1.823,-1.810,1.707,-1.248,1.561,-1.859,1.940,-1.229)$

Therefore, a console application program TROMBONE was upgraded using 9 independently adjusted qudrupoles. 3 expected tune changes set in the console and the respective Schottky spectrum are shown in Fig.4 We can see that the difference between expected and measured tune change is about 0.001 .
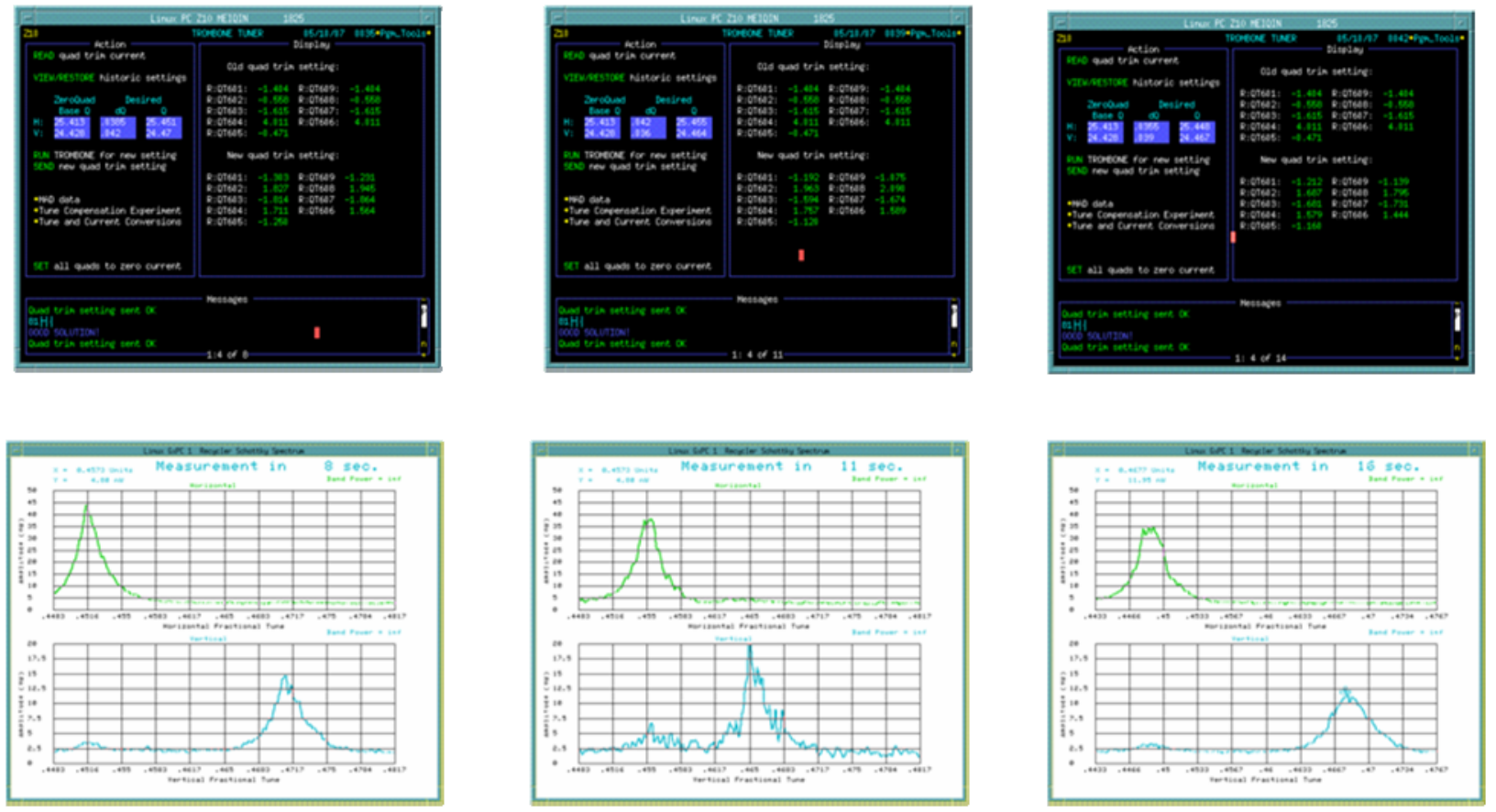

Expected: $(0.451,0.470)$ Measured: $(0,4616,0,4709)$

Expected: (0.465, 0.464) Measured: $(0.4550,0.4654)$

Expected: $(0.448,0.467)$ Measured: $(0,4485,0.4654)$

Fig. 4 Console tune settings and the respective Schottky spectrum

\section{Lattice measurement after TROMBONE program upgraded}

Differential orbits of 24 correctors were taken at the tunes set to $(0.4578,0.4656)$, and LOCO (Linear Optics from Closed Orbit) fittings were done with the rest of the orbit data. Fig.5 shows the orbit before and after LOCO fittings. Beta-functions obtained from this measurement are shown in Fig.6. We can see that the beta-waves are significantly reduced now in the Recycler ring, compared to those in Fig. 2 

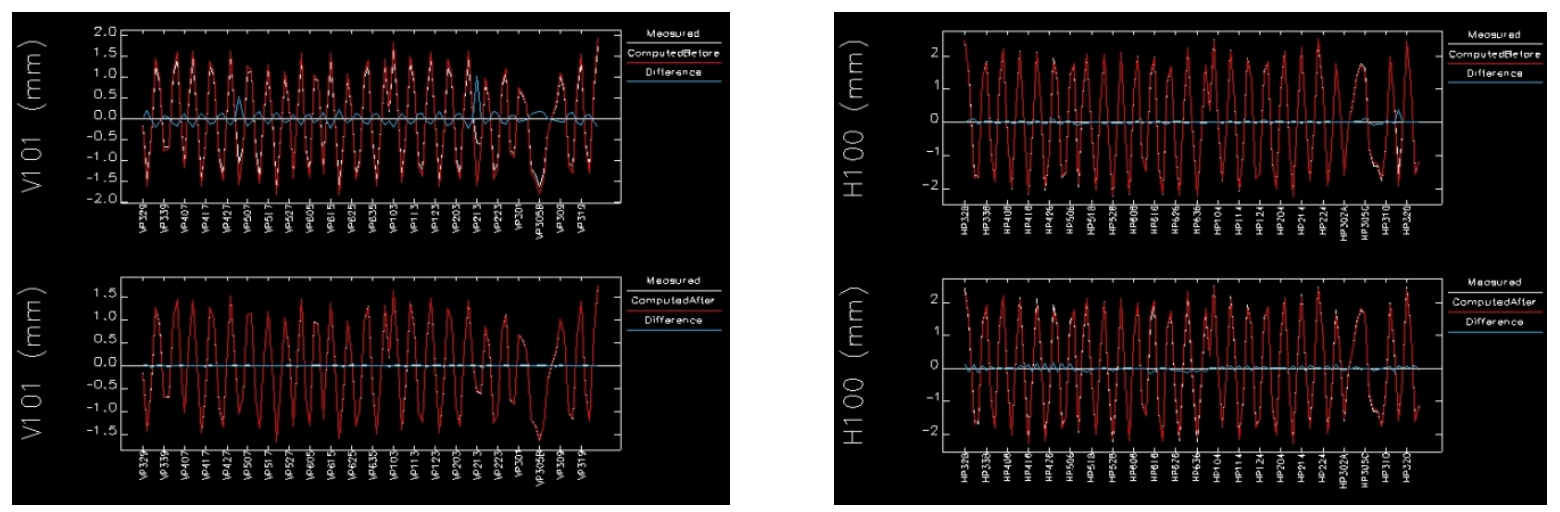

Fig 5 orbits before and after LOCO fittings

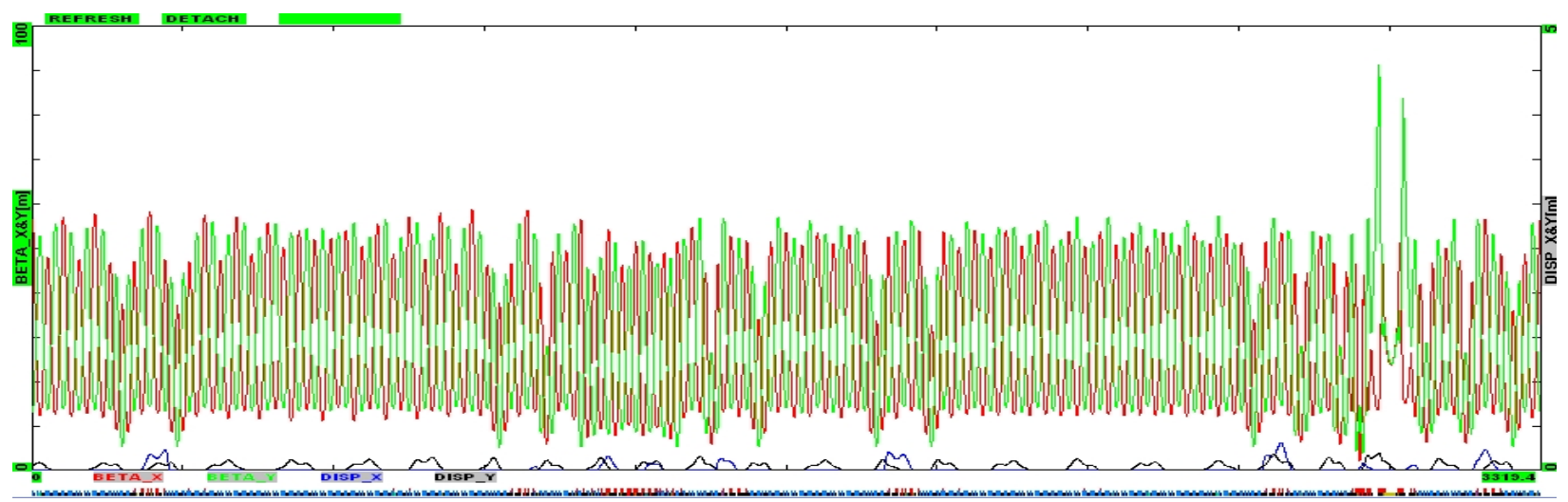

Fig. 6 Beta-functions obtained from differential orbit measurement at working point of $(25.455,24.464)$ after TROMBONE program ungraded.

\section{Extend Trombone tuning range}

It was asked to explore beam's performance at the lower end of tune diagram if the intensity of the stored pbars is increased to $600 \mathrm{E} 10$. However, maximum tune changes can only be approximately $\Delta v_{x}=-0.06$, $\Delta v_{\mathrm{y}}=-0.18$ with the limit of qudrupole current up to 6.5 Amperes. Several suggestions were proposed. With the upgraded program, it was found that if the given tune change is $\Delta v_{\mathrm{x}}=-0.2, \Delta v_{\mathrm{y}}=-0.2$, the current of 9 quadrupole circuits would be in:

$\mathrm{I} 1, \mathrm{I} 2, \ldots, \mathrm{I} 9=(5.984,-9.308,8.900,-8.762,6.018,-8.007,8.810,-9.913,6.225)$

If we install one more pair of trim quads at the existing location, the current of each circuit will be brought down to less than5.0 Amperes. It would be working for us to adjust the tunes down to $(.20 \sim .25)$ region, and it would be easiest way to extend trombone tuning range.

During 2007 Summer Shutdown, two additional quadrupoles were installed at each pair of the existing trim quads location, shown in Fig.7 


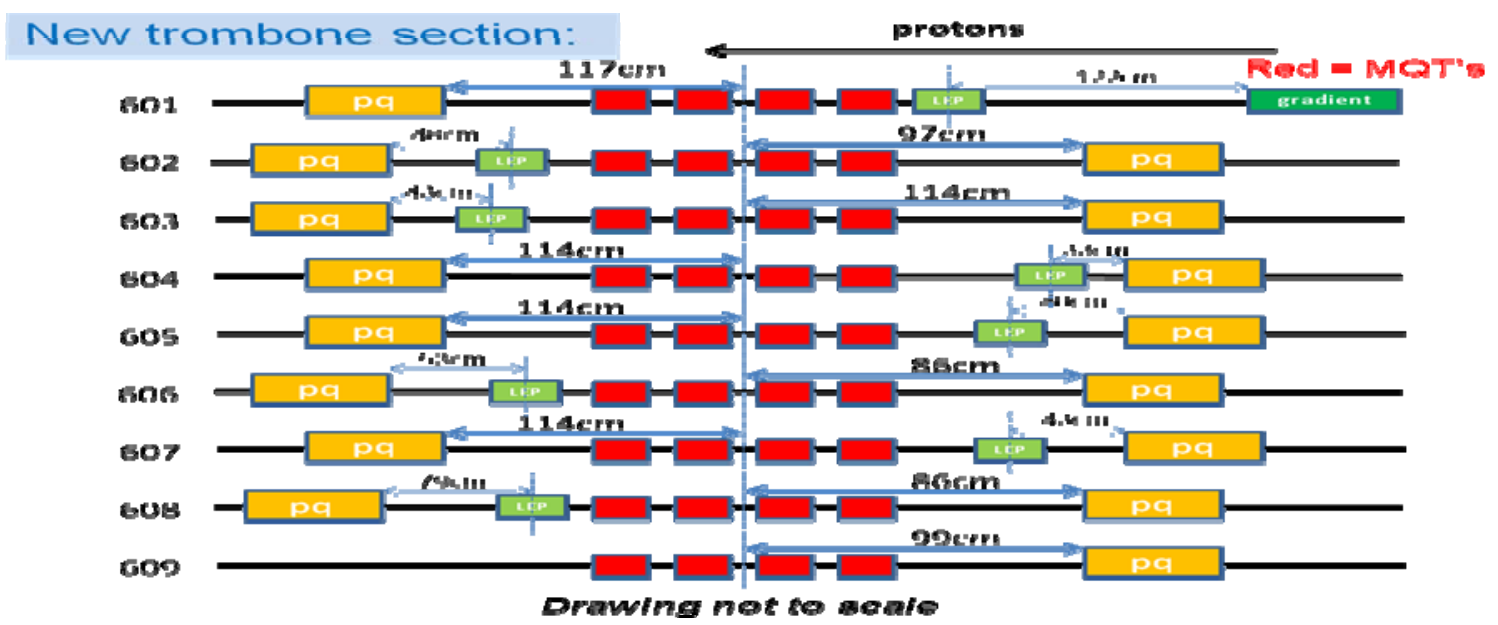

Fig.7 New trombone section from MRK601 to MRK609.
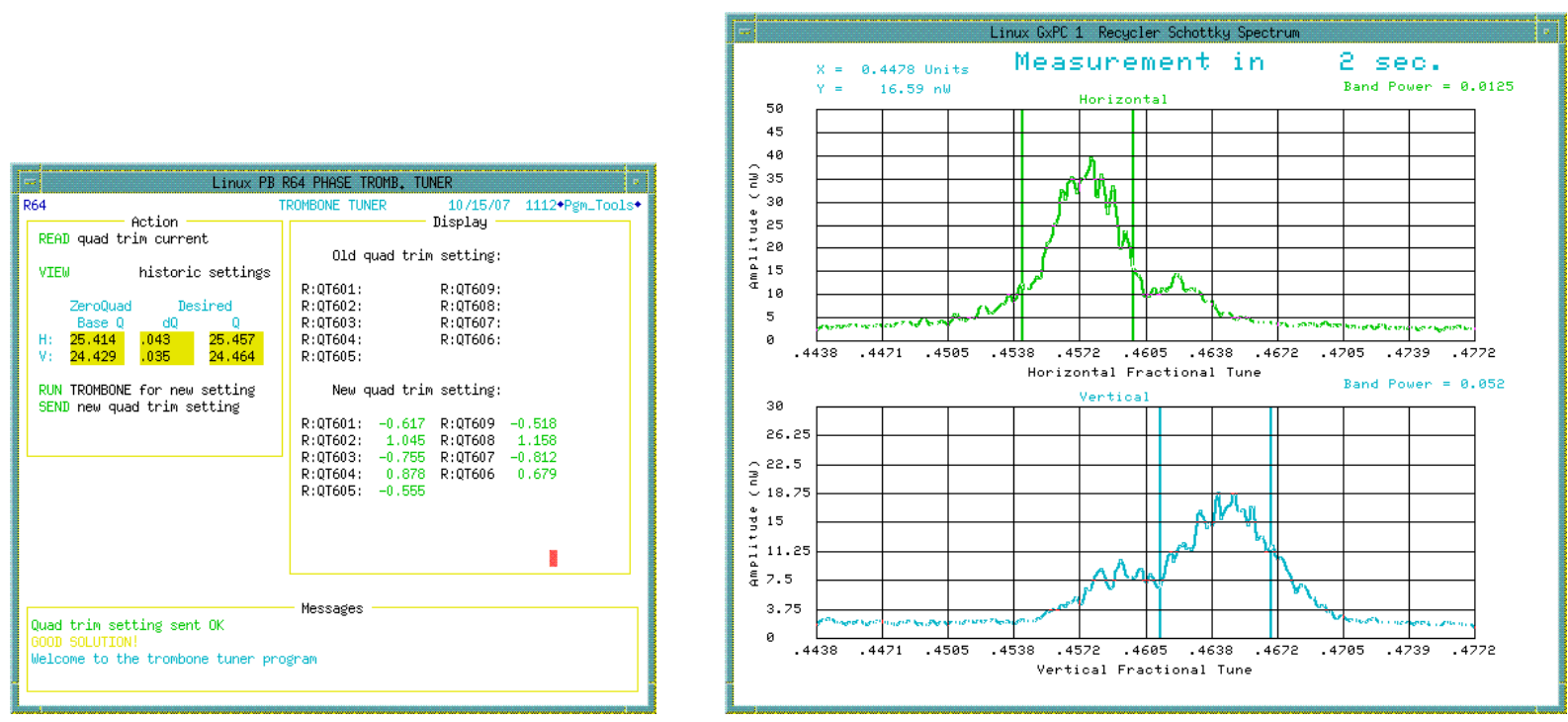

Fig 8. The expected tunes and the measured tunes (the respective Schottky spectrum)

We updated Recycler lattice for code OPTIM and MAD with the new trombone structure, and modified the MATHEMATICA program so that it accommodated the new trombone structure. The general equations with new trombone structure are as follows:

$\left(\begin{array}{ccccccccc}-140.38 & 1688.13 & 187.477 & -1449.79 & -74.7654 & 1810.12 & 104.737 & -1521.67 & -49.9376 \\ 33.8252 & 68.4684 & -35.4021 & -50.3818 & 36.5726 & 37.2133 & -37.8437 & -26.0246 & 35.9651 \\ 5255.4 & -1397.95 & -3860.68 & 1655.56 & 2661.18 & -1612.78 & -1038.58 & 1731.92 & 267.428 \\ -156.345 & -21.1427 & 170.255 & 12.4532 & -181.473 & -11.8655 & 188.58 & 6.90345 & -191.288 \\ 2.81678 & 16.2875 & 3.08217 & 14.2611 & 2.8848 & 16.3927 & 3.05359 & 14.1942 & 2.82223 \\ -15.3701 & -2.84064 & -15.1444 & -2.91699 & -15.178 & -2.83305 & -15.182 & -2.94246 & -15.1916\end{array}\right)\left(\begin{array}{l}k_{1} \\ k_{2} \\ k_{3} \\ k_{4} \\ k_{5} \\ k_{6} \\ k_{7} \\ \boldsymbol{k}_{8} \\ \boldsymbol{k}_{9}\end{array}\right)=\left(\begin{array}{c}-0.104264 \\ 0.018209 \\ -0.173774 \\ 0.00289952 \\ \Delta \mu_{x} \\ \Delta \mu_{y}\end{array}\right)$


Consol application program TROMBONE was also modified for the new trombone structure, one of the tune settings and the respective Schottky spectrum are shown in the Fig 8.

Differential orbits of 48 correctors were taken at the tunes set to current working point $(0.4593,0.4657)$ and $(0.4146,0.4299)$, which is corresponding to the settings of zero current of 9 groups of the quadrupoles. LOCO (Linear Optics from Closed Orbit) fittings were done with all the orbit data.. Betafunctions obtained from orbit measurement for the Recycler with new trombone structure at current working point and with Zero trombone quad settings are shown in Fig.9 and Fig. 10.

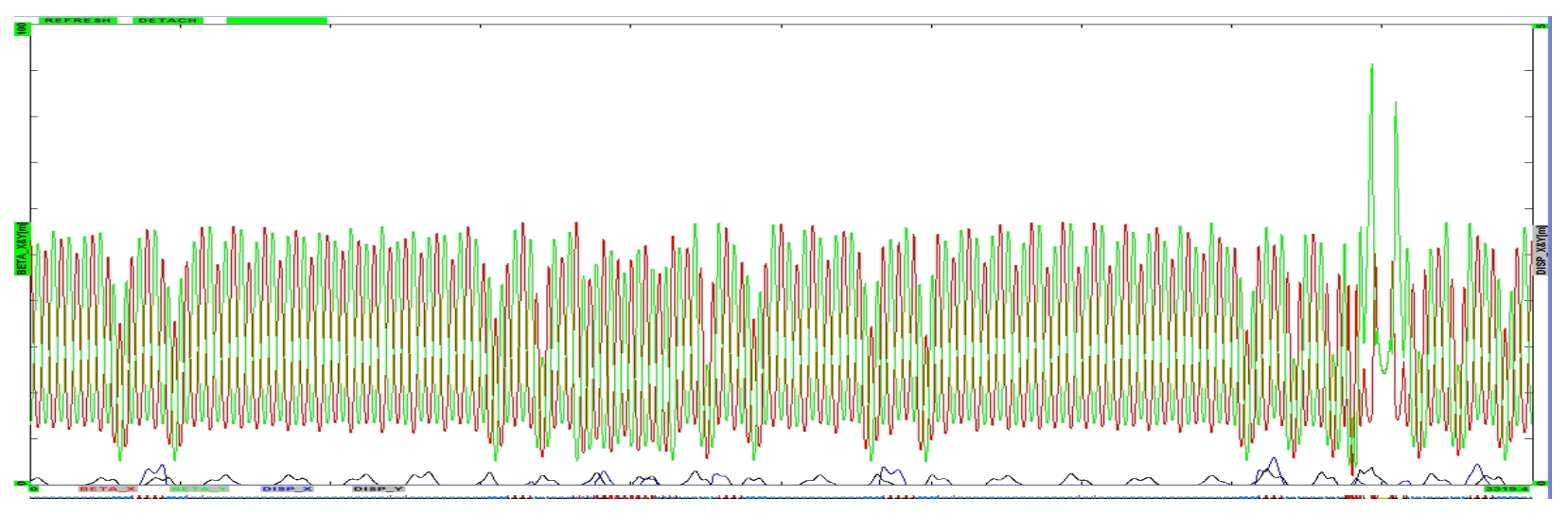

Fig. 9 Beta-functions with new TROMBONE structure at current working point $(25.4593,24.4657)$

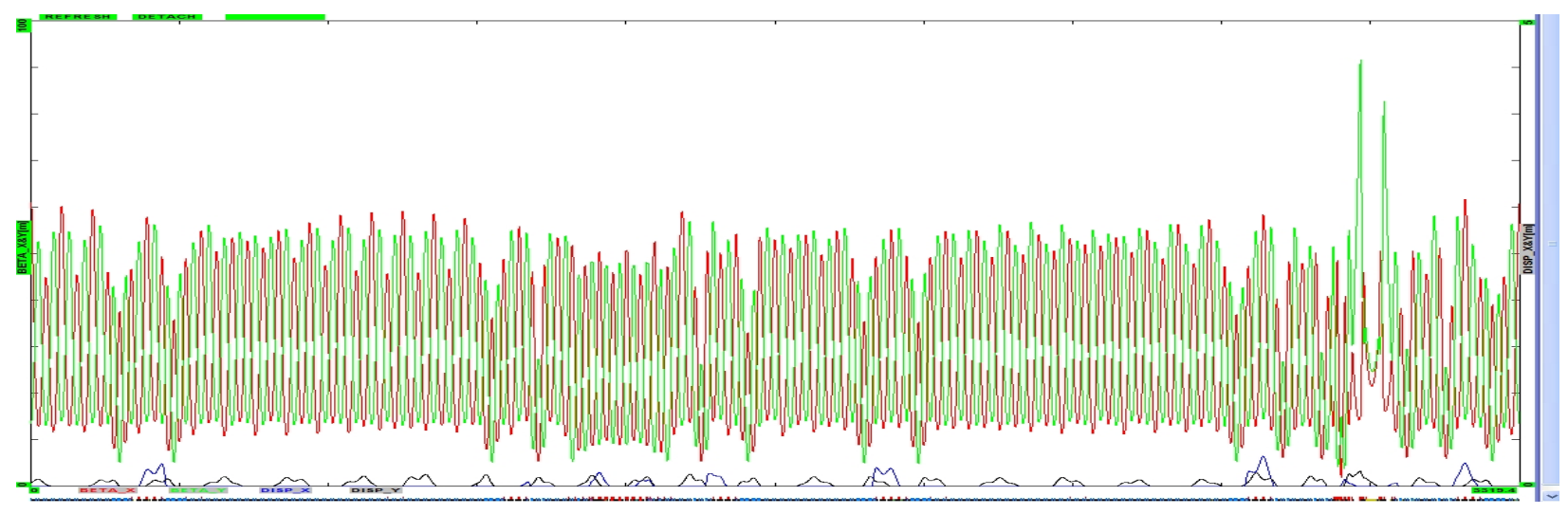

Fig. 10 Beta-functions with new TROMBONE structure at current working point at $(0.4145,0.4299)$

\section{Life time vs. tunes in the region of 0.2-0.25 of tune diagram}

We did lifetime scanning in the region of $0.2-0.25$ of tune diagram. Fig 5 shows the current working points and the scanning points in the tune diagram of the Recycler ring. The procesure to measure the lifetime was as follows:

(1) Inject beam and debunch;

(2) Move a horizontal and a vertical jaw into the acceptance of the machine (and beam) so that emittance growth rates due to tunes will directly cause a change in lifetime quickly. A recommended set of values is: $\mathrm{R}: \mathrm{WALLJ}=10 \mathrm{~mm}$, R:TOPJ $=9.5 \mathrm{~mm}$.

(3) Measure lifetime with D44 for at least 15 minutes using exponential fitting. 
(4) To change tunes, move the jaws back a few millimeters; use console application program TROMBONE to change tunes to new targets, and move the jaws back in to limit acceptance.

We have checked the closed orbit at lower tunes, and compared it with the closed orbit at current working point tunes, however, we should have also verified that the default orbit (with minimal sextupole and quadrupole feed down) is intact. The reason we did not do it was that we didn't want to mess up the corrector's setting for our present operation conditions).

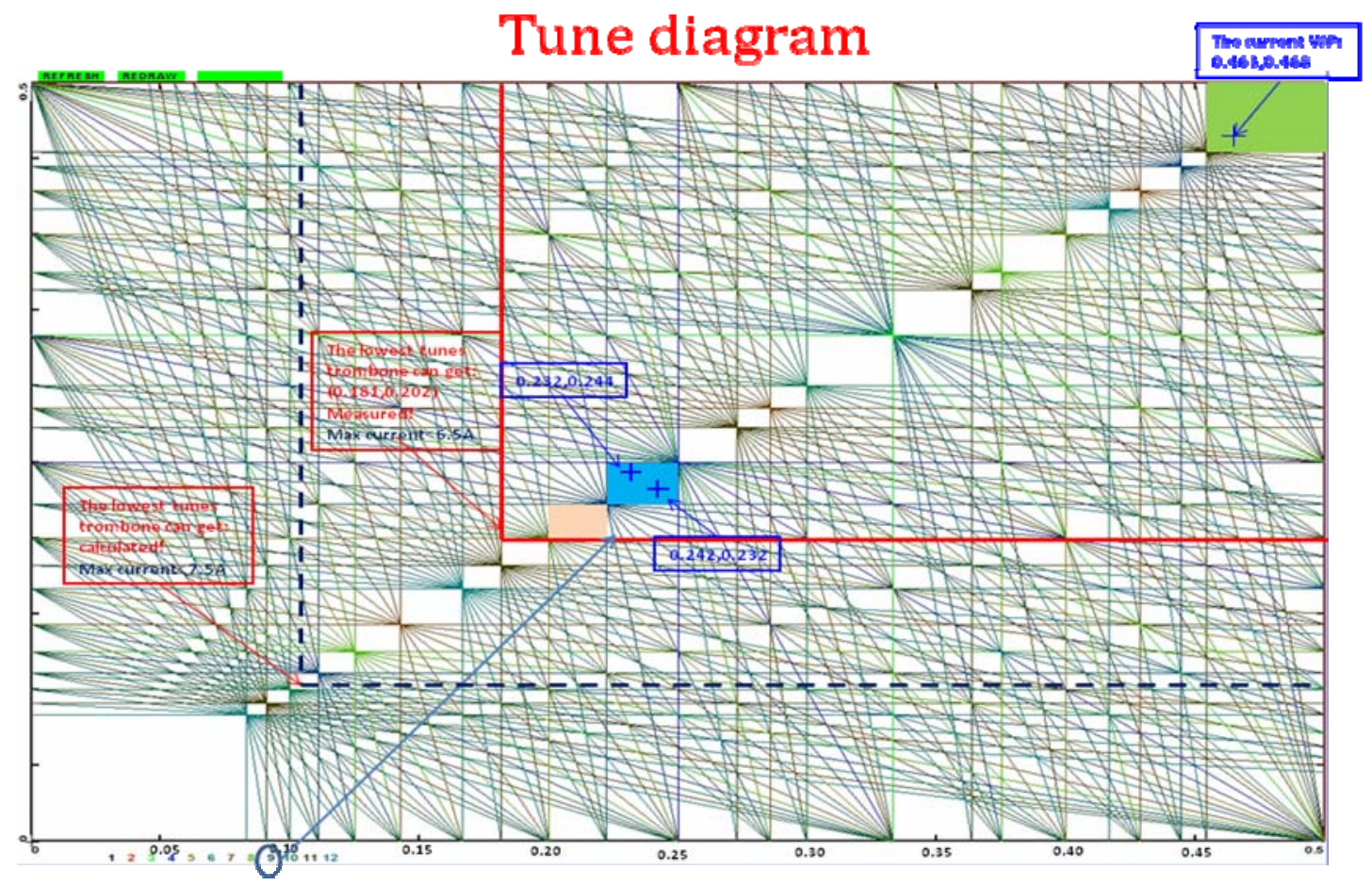

It's found that there is about $10 \%$ deviation of measured tunes from expected tunes at lower tune region, which need larger current settings of trim qaudrupoles. It must be resulted in the linear approximations in obtaining linear equation. It needs to find a way to correct this deviation. Since the study time was limited, we only scanned 3 points in tune diagram. The lifetime obtained by the way we scanned was 3.0 Hrs at current working point $(0.463,0.468)$ and about $2.1 \mathrm{hrs}$ at both $(0.232,0.244)$ and $(.242,232)$. It did not show too much difference in lifetime, although the closed orbit at lower tunes was not smoothed well. 
The deviation of measured tunes from expected

(base tunes:0.414,0.430)

\begin{tabular}{|c|c|c|c|c|c|c|c|}
\hline \multicolumn{2}{|c|}{$\begin{array}{l}\text { Expected } \\
\text { (Mathematica) }\end{array}$} & \multicolumn{3}{|c|}{ Optim gives } & \multicolumn{2}{|c|}{ Measured } & \multirow{2}{*}{$\begin{array}{c}\text { Deviation } \\
\text { from expected }\end{array}$} \\
\hline $\begin{array}{l}\mathrm{Q}_{\mathrm{x}}, \\
\mathrm{Q}_{\mathrm{y}}\end{array}$ & $\begin{array}{l}\mathrm{DQ}_{\mathrm{x}} \\
\mathrm{DQ}_{\mathrm{y}}\end{array}$ & $\begin{array}{l}\mathrm{Q}_{\mathrm{x}} \\
\mathrm{Q}_{\mathrm{y}}\end{array}$ & $\begin{array}{l}\mathrm{DQ}_{\mathrm{x}} \\
\mathrm{DQ}_{\mathrm{y}}\end{array}$ & $\begin{array}{c}\text { Deviation } \\
\text { from expected }\end{array}$ & $\begin{array}{l}\mathrm{Q}_{\mathrm{x}} \\
\mathrm{Q}_{\mathrm{y}}\end{array}$ & $\begin{array}{l}\mathrm{DQ}_{\mathrm{x}} \\
\mathrm{DQ}_{\mathrm{y}}\end{array}$ & \\
\hline $\begin{array}{l}0.164 \\
0.18\end{array}$ & $\begin{array}{l}-0.25 \\
-0.25\end{array}$ & $\begin{array}{l}0.181 \\
0.200\end{array}$ & $\begin{array}{l}-0.233 \\
-0.230\end{array}$ & $\begin{array}{l}0.017 \\
0.022\end{array}$ & $\begin{array}{l}0.186 \\
0.207\end{array}$ & $\begin{array}{l}-0.228 \\
-0.223\end{array}$ & \\
\hline $\begin{array}{l}0.218 \\
0.223\end{array}$ & $\begin{array}{l}-0.186 \\
-0.207\end{array}$ & $\begin{array}{l}0.241 \\
0.235\end{array}$ & $\begin{array}{l}-0.173 \\
-0.195\end{array}$ & $\begin{array}{l}0.013 \\
0.012\end{array}$ & $\begin{array}{l}0.232 \\
0244\end{array}$ & $\begin{array}{l}-0.182 \\
-0.186\end{array}$ & \\
\hline $\begin{array}{l}0.225 \\
0.210\end{array}$ & $\begin{array}{l}-0.189 \\
-0.22\end{array}$ & $\begin{array}{l}0.239 \\
0.222\end{array}$ & $\begin{array}{l}-0.175 \\
-0.208\end{array}$ & $\begin{array}{l}0.014 \\
0.012\end{array}$ & $\begin{array}{l}0.242 \\
0.232\end{array}$ & $\begin{array}{l}-0.172 \\
-0.198\end{array}$ & \\
\hline $\begin{array}{l}0.463 \\
0.468\end{array}$ & $\begin{array}{l}0.049 \\
0.038\end{array}$ & $\begin{array}{l}0.466 \\
0.467\end{array}$ & $\begin{array}{l}0.052 \\
0.037\end{array}$ & $\begin{array}{l}0.003 \\
0.001\end{array}$ & $\begin{array}{l}0.463 \\
0.466\end{array}$ & $\begin{array}{l}0.049 \\
0.036\end{array}$ & \\
\hline $\begin{array}{l}0.462 \\
0.473\end{array}$ & $\begin{array}{l}0.048 \\
0.043\end{array}$ & $\begin{array}{l}0.465 \\
0.472\end{array}$ & $\begin{array}{l}0.051 \\
0.042\end{array}$ & $\begin{array}{l}0.003 \\
0.001\end{array}$ & $\begin{array}{l}0.462 \\
0.473\end{array}$ & $\begin{array}{l}0.048, \\
0.043\end{array}$ & \\
\hline
\end{tabular}

\section{Conclusion}

The Recycler lattice has been measured and corrected to what we expected one with little beta-wave. The trombone tuning range was extended to reach the lowest tune of 0.2 in both planes by installing one more pair of the trim quads. The console application program has been upgraded several times according the trombone structure, and it works well in operation now.

\section{Acknowledgement}

I'd like to thank Valdmir Nagaslaev for his program to take differential orbit data, and Alexander Valishev for his help with the LOCO fitting. Many thanks to Martin $\mathrm{Hu}$ for his help with the measurement of the tune spectrum for the lifetime. 


\section{References}

[1] M. Xiao, et.al, "Linear Lattice Modeling of the Recycler ring at Fermilab". Proceedings of EPAC 2005, Edinburgh, Scotland. P2056-2058;

[2] Geery Jackson, "The Fermilab Recycler Ring Technical Design Report"Fermilab-TM-1991, November 1996;

[3] M. Xiao, "Phase Trombone Program Migration for the Recycler Ring at Fermilab". Proceedings of 2005 PAC, Knoxville, Tennessee. P3135-3137; 